Results The subjects with hypertension reported significant higher effort, over commitment, and ERI, while lower reward. Among men, high effort, high over commitment and high ERI increased risk of presence of hypertension, with odd ratios (OR) of 10.0 (95\% CI 5.8 to 17.3 ), 5.0 (95\% CI 3.0 to 8.2 ) and 11.1 ( $95 \%$ CI 5.7 to 21.6 ), respectively, compared with the low level of job stress. After adjustment for age, BMI, dyslipidemia and diabetes mellitus, the associations were still significant, with an adjusted OR of 4.6 (95\% CI 2.5 to 8.5), 5.0 (95\% CI 2.7 to 9.2 ) and 8.2 (95\% CI 3.9 to 17.1), for effort, over commitment, and ERI, respectively. High reward played as a protective factor with OR of 0.3 (95\% CI 0.2 to 0.6 ), and adjusted OR of 0.2 (95\% CI 0.1 to 0.5$)$, compared with low level of reward. Among women, the results were similar, and the adjusted $\mathrm{OR}$ was 3.3 (95\% CI 1.2 to 8.9$), 4.1$ (95\% CI 1.4 to 11.8$), 3.8(95 \%$ CI 1.3 to 10.7$)$ and 0.2 (95\% CI 0.1 to 0.7$)$ for effort, over commitment, ERI and reward, respectively.

Conclusion Job stressors such as high effort, over commitment, ERI increased risk of hypertension and high reward reduced risk of hypertension among Chinese workers. It is of great interest to understand and improve job stress among Chinese workers for the purpose of prevention of hypertension. Further studies in larger sample size and in prospective design will be needed to confirm the role of job stressors on hypertension among Chinese workers.

\section{[gw22-e0658] JOB STRESS ASSOCIATED WITH BLOOD PRESSURE AMONG CHINESE WORKERS: RESULTS FROM SHISO STUDY}

Weixian Xu', Juan Hang'1, Wei Gao², Yiming Zhao², Lijun Guo ${ }^{1}$ Peking University Shenzhen Hospital, Guangdong, China; ${ }^{2}$ Peking University Third Hospital, Beijing, China

\subsection{6/heartjnl-2011-300867.299}

Objective Relevant studies reported that job stress played an important role on hypertension. However, previous studies have produced mixed findings because of the use of different job stress questionnaires and different populations. The knowledge about the effect of job stress on blood pressure is rare among Chinese population. The aim was to explore the association between job stressors, such as effort, reward, over commitment, effort-reward imbalance (ERI) and blood pressure among Chinese workers.

Methods The Stress and Health in Shenzhen Workers (SHISO) study was a cross-sectional study which aimed to evaluate the impact of job stress on physical health in Shenzhen workers. A total of 881 took part in this SHISO study. The authors excluded subjects who had a history or new detected diseases at the medical checkup including heart disease, or peripheral arterial or cerebrovascular diseases, and participants with incomplete questionnaires. The final sample was 734 subjects (508 men and 226 women). Simplified Chinese version of effort-reward imbalance questionnaire (ERIQ) was used to evaluate job stress level. Blood pressure (BP) was taken by standard and periodically calibrated mercury sphygmomanometers in the sitting position by the trained technicians. The authors defined hypertension as systolic blood pressure (SBP) $\geq 140 \mathrm{~mm} \mathrm{Hg}$ or diastolic blood pressure (DBP) $\geq 90 \mathrm{~mm} \mathrm{Hg}$ or both, or use of blood pressure lowering medication, or hypertension history with a physician's diagnosis certificate. 